\title{
Leukocyte Adhesion-deficient Neutrophils Fail to Amplify Phagocytic Function in Response to Stimulation Evidence for CD11b/CD18-dependent and -independent Mechanisms of Phagocytosis
}

\author{
Hattie D. Gresham, * Irene L. Graham, ${ }^{\star}$ Donald C. Anderson," and Eric J. Brown \\ *Department of Pharmacology, University of Missouri-Columbia, Columbia, Missouri 65212; Department of Pediatrics, \\ Baylor College of Medicine, Houston, Texas 77030; and ${ }^{\ddagger}$ Departments of Medicine, Cell Biology, and Physiology, \\ and Microbiology and Immunology, Washington University, St. Louis, Missouri 63110
}

\begin{abstract}
Stimulation of PMN with inflammatory mediators markedly augments Fc and CR1 receptor-mediated ingestion. However, CD11/CD18-deficient PMN from three patients with complete leukocyte adhesion deficiency (LAD) failed to recruit phagocytic function in response to phorbol esters, cytokine, or ArgGly-Asp-containing ligand stimulation. Because stimulated ingestion is protein kinase $C$ (PKC)-dependent, our data indicate that LAD PMN exhibit only PKC-independent phagocytosis. The defect in PKC-dependent ingestion is specific for CD11b/ CD18 and not secondary to the chronic or recurrent infections which occur in this disease. The LAD phenotype for phagocytic function can be reproduced in normal PMN by the anti-CD11b MAbs OKM1 and OKM10. In contrast, MAb Mo1 (antiCD11b) and MAb IB4 (anti-CD18) inhibit both CD11b/ CD18-dependent and -independent mechanisms of ingestion by normal PMN. Their ability to inhibit CD11b/CD18-independent ingestion may be mediated by CAMP, as shown by experiments with a protein kinase A inhibitor $\mathrm{HA1004}$ and by direct measurement of cAMP levels in immune complex- and FMLPstimulated PMN. These data indicate that CD11 b/CD18-independent and -dependent mechanisms of phagocytosis exist and that some effects of anti-CD11b/CD18 MAbs may be mediated by alterations in cAMP levels. (J. Clin. Invest. 1991. 88:588-597.) Key words: CD116/CD18 • cyclic adenosine monophosphate $\bullet$ phagocytosis
\end{abstract}

\section{Introduction}

Phagocytosis is a complex biological process of specialized cells which is essential for normal host defense. It is necessary not only for the uptake and destruction of pathogens but also for the clearance of immune complexes, removal of damaged tissue, and initiation of wound repair. Despite its central role in host defense, knowledge of the regulation of phagocytic function at inflammatory sites has lagged behind understanding of activation of the respiratory burst and exocytosis, other func-

This work was presented in part at the annual meeting of the American Federation for Clinical Research, Washington, DC, May 1989, and also appeared in abstract form (1989. Clin. Res. 37:564A).

Address reprint requests to Dr. Gresham, Room 517B, 1 Hospital

Drive, University of Missouri-Columbia, Columbia, MO 65212.

Received for publication 30 July 1990 and in revised form 28 February 1991.

J. Clin. Invest.

(c) The American Society for Clinical Investigation, Inc.

0021-9738/91/08/0588/10 \$2.00

Volume 88, August 1991, 588-597 tions of professional phagocytes required for the maintenance of homeostasis. Recent investigations from our group into the regulation of human neutrophil (PMN) phagocytosis have led us to hypothesize that phagocytosis by PMN is primarily a recruited function at inflammatory sites (1-5). Stimulation of PMN with various inflammatory mediators markedly augments both Fc- and CR1-mediated ingestion (1-9). This recruitment of phagocytic function is reflected not only in the total number of ingested targets but also in the percentage of PMN participating in the phagocytic process $(1,2)$. Presumably, this regulation of ingestion acts to limit the damaging products of PMN activation, such as toxic oxygen metabolites and secreted lysosomal products, to the site of inflammation.

We and others have found that PMN can recruit several distinct molecular mechanisms for phagocytosis in response to inflammatory signals, which may be regulated further by the specific opsonin (IgG, C4b, C3b, and C3bi) and opsonin receptor (FcRII, FcRIII, CR1, and CR3) engaged (1-9). In addition, monocytes and macrophages employ quite different molecular mechanisms for phagocytosis from those employed by PMN, even when the same opsonin or opsonin receptor is involved $(1,10)$. In this manner, at inflammatory sites where PMN and macrophages encounter pro-inflammatory cytokines, interferons, interleukins, chemotactic peptides, and components of the extracellular matrix, ingestion is not only augmented, but the molecular mechanism involved in phagocytosis may be altered. As an example, we have shown recently that ingestion of IgG-opsonized targets by nonstimulated PMN is not dependent upon protein kinase $\mathrm{C}(\mathrm{PKC})^{1}$ activation. However, when PMN are stimulated with tumor necrosis factor- $\alpha$ (TNF- $\alpha$ ), not only is ingestion of the IgG-opsonized targets markedly increased, but PKC translocation is also greatly augmented over the minimal amount observed with either IgG or TNF- $\alpha$ alone (11). This synergism in activation of PKC is essential for the increased ingestion by PMN exposed to TNF- $\alpha$ (11). The importance of these data to host defense is demonstrated by the fact that PMN from patients with chronic granulomatous disease (CGD), which lack the ability to generate a respiratory burst, as well as PMN from patients with paroxysmal nocturnal hemoglobinuria, which lack expression of FcRIII, fail to amplify ingestion of IgG-opsonized targets in response to cytokine (TNF- $\alpha$ ) stimulation $(4,12)$. Therefore, defects in the different

1. Abbreviations used in this paper: $\mathrm{CGD}$, chronic granulomatous disease; DiBcAMP, dibutyrl cAMP; E, sheep erythrocyte(s); EC4b and EIgG, sheep erythrocyte(s) opsonized with C4b or IgG; respectively; Fn, fibronectin; IBMX, isobutylmethylxanthine; LAD, leukocyte adhesion deficiency; PDBu, phorbol dibutyrate; PI, phagocytic index; PKA and PKC, protein kinase A and C; RGD, Arg-Gly-Asp; TNF- $\alpha$, tumor necrosis factor- $\alpha ; \mathrm{Vn}$, vitronectin. 
molecular mechanisms for phagocytosis employed by PMN at inflammatory sites may be observed in both genetic and acquired disorders which exhibit a predisposition to serious and recurrent infection.

In this regard, the purpose of the present work is to determine whether CD1 1/CD18-deficient PMN from three patients with complete leukocyte adhesion deficiency (LAD) (13) would be capable of recruiting the ingestion mechanisms expected to predominate at inflammatory foci. These patients lack the expression of a family of adhesion molecules (CD11a,b,c/CD18) owing to deficient production or maturation of the common $\beta$ chain of these heterodimers (13). In vivo, this defect results in a predisposition to severe recurrent bacterial infections (14). The PMN from patients with LAD are thought to exhibit normal phagocytic function in the absence of stimulation, as long as the target is opsonized with a ligand for a receptor other than CD1 1b/CD18, (e.g., IgG) (14). However, we and others have shown previously that some monoclonal antibodies (MAbs) to either CD11b (Mo1) or to CD18 (1B4) inhibit both stimulated and nonstimulated ingestion of targets opsonized with non-CD11b/CD18 ligands (15-17). We have interpreted these data to mean that $C D 11 \mathrm{~b} / \mathrm{CD} 18$ plays a role in the regulation of both stimulated and nonstimulated phagocytosis independent of its ligand-binding function. The purpose of the present work is to resolve this apparent discrepancy.

One possibility to reconcile these data is that the antibodies to $\mathrm{CD} 11 \mathrm{~b} / \mathrm{CD} 18$ that inhibit ingestion do so by generating an intracellular signal which is inhibitory for ingestion. Alternatively, the patients' PMN may have compensatory mechanisms of ingestion that are in fact different from the mechanisms used by normal PMN. Our extensive investigations into the phagocytic mechanisms employed by normal PMN afforded us the opportunity to assess if the same mechanisms are used by LAD PMN. In the present work, we show that PMN from patients with LAD fail to amplify ingestion for either IgGor $\mathrm{C} 4 \mathrm{~b}$-opsonized targets in response to stimulation by phorbol esters, cytokines, or Arg-Gly-Asp (RGD)-containing adhesive proteins. Whereas LAD PMN are defective in the mechanism for stimulated ingestion, our data indicate that they do possess and use the mechanism of unstimulated IgG-dependent ingestion used by normal PMN. In addition, our data indicate that the monoclonal antibodies to CD1 1b, OKM1, and OKM10 do not inhibit unstimulated ingestion but do inhibit stimulated ingestion. In this manner, treatment of normal PMN with these antibodies to $\mathrm{CD} 11 \mathrm{~b}$ results in reproduction of the LAD phenotype for phagocytic function. In order to explain why antibodies Mol and 1B4 inhibit a CD11/CD18-independent mechanism of phagocytosis, we investigated their effects on the intracellular levels of cyclic adenosine monophosphate (cAMP). We had shown previously that agents that increase intracellular cAMP inhibit unstimulated but not stimulated ingestion (3). We present data which indicate that Mol and 1B4, but not control antibodies, augment the accumulation of cAMP in response to either immune complex or $N$-formylmethionyl-leucyl-phenylalanine (FMLP) stimulation. In addition, the ability of these antibodies to inhibit unstimulated ingestion but not stimulated ingestion is abrogated by the inclusion of HA1004, a putative protein kinase A (PKA) inhibitor. Thus, in the presence of a PKA inhibitor, Mol and 1B4 can also reproduce the LAD phenotype for phagocytic function.

These data indicate that at inflammatory sites, augmentation of PMN phagocytic function is absolutely dependent on the CD1 1b/CD18 complex. Because we believe that the mechanism for stimulated ingestion is essential for normal host defense, this suggests that the susceptibility to infection in patients with LAD may be related in part to a failure to enhance ingestion in response to inflammatory stimuli. This may be particularly important at sites, such as the lung, where CD11/ CD18-deficient PMN ingress in response to inflammation is relatively normal. Finally, our data demonstrate that both CD11b/CD18-independent and -dependent mechanisms of phagocytosis exist in PMN. The ability of antibodies Mol and IB4 to inhibit the CD11/CD18-independent mechanism of ingestion may be explained by their ability to augment increases in intracellular cAMP in response to stimulation. This suggests that other effects of some antibodies and potential ligands for the CD11b/CD18 complex may result from alterations in cellular cAMP metabolism.

\section{Methods}

Special reagents. The following reagents were purchased from Sigma Chemical Co., St. Louis, MO: phorbol dibutyrate (PDBu), phorbol myristate acetate (PMA), FMLP, isobutylmethylxanthine (IBMX), catalase (bovine liver, 52,000 U/mg), superoxide dismutase (bovine erythrocytes, 3,000 U/mg), cytochrome $c$ (type VI, horse heart), and dibutyrl cAMP (DiBcAMP). PDBu $(1 \mathrm{mg} / \mathrm{ml})$, phorbol myristate acetate (PMA, $1 \mathrm{mg} / \mathrm{ml})$, FMLP $(22.9 \mathrm{mM})$, and IBMX $(80 \mathrm{mM})$ were prepared as stock solutions in (DMSO, Aldrich Chemical Co., Milwaukee, WI), were stored at $-70^{\circ} \mathrm{C}$, and were diluted into aqueous medium immediately before use. Equivalent concentrations of vehicle were used to control for solvent effects. Chicken egg albumin (ovalbu$\mathrm{min}$ ) and human vitronectin ( $\mathrm{Vn}$ ) were purchased from CalbiochemBehring Corp., La Jolla, CA. The isoquinolinesulfonamide derivatives H7 and HA1004 were purchased from Seikagaku America, St. Petersburg, FL. They were reconstituted $(10 \mathrm{mM})$ with phosphate-buffered saline and stored protected from light at $4^{\circ} \mathrm{C}$. A 10 -fold concentrated stock of Hanks' balanced salt solution (HBSS) was purchased from Gibco Laboratories, Grand Island, NY. Human fibronectin (Fn) was purified as described (18). The synthetic peptide GRGDSC was prepared by the Protein Chemistry Facility, Washington University School of Medicine. GRGDSC and $C$ as a control were linked to pigeon heart cytochrome $c$ as described (5).

MAbs. Purified MAb to CD11b/CD18 were obtained as follows: OKM1 and OKM10 (anti-CD1 1b) were the generous gifts of Dr. Patricia Rao, the R. W. Johnson Pharmaceutical Research Institute, Raritan, NJ; Mo-1 (IgG2a anti-CD11b) and IB4 (IgG2a anti-CD18) as described (17), Leu-15 (IgG2a anti-CD1 1b) from Becton, Dickinson \& Co., San Jose, CA; and isotype controls 4B2 (IgG2b) and 1B5 (IgG2a) as described (2). 4B2 and 1B5 recognize unknown antigens expressed on PMN. Purified murine monoclonal anti-FcRI (32.2), anti-FcRII (IV.3), and anti-FcRIII (3G8) were purchased from Medarex, Inc., West Lebanon, NH. Anti-CR1 (3D9) was obtained as described (7).

Isolation of PMN. PMN were isolated as described (3) from normal volunteers, three patients with LAD followed at the Baylor College of Medicine (14), and two patients with CGD followed at the University of Missouri-Columbia School of Medicine. Patient samples were obtained when patients were free from infection. LAD patient blood samples were always accompanied by blood drawn at the same time from a normal control. The PMN from these normal volunteers served to control for any effects of transportation on PMN function. Multiple studies of the normal transported PMN showed that their phagocytic, respiratory burst, and excretory responses were not different from PMN isolated from normal volunteers from the laboratory. The LAD patients have been studied previously (14) and their PMN were shown then and subsequently by us to lack antigenic expression of CD18 as assessed by MAb IB4 positivity measured by fluorescent flow microcytometry (5). Unless indicated otherwise, PMN were suspended in HBSS containing $4.2 \mathrm{mM} \mathrm{NaHCO}_{3}, 10 \mathrm{mM}$ Hepes, $1.5 \mathrm{mM} \mathrm{CaCl}_{2}, 1.5$ $\mathrm{mM} \mathrm{MgCl} 2$, and $1 \%$ ovalbumin, pH $7.4\left(\mathrm{HBSS}^{++}-1 \% \mathrm{OVA}\right)$. 
Phagocytic targets. Sheep erythrocytes (E) were purchased from Whittaker M.A. Bioproducts, Walkersville, MD. IgG (EIgG and $\mathrm{ElgG}_{\mathrm{hi}}$ )- and $\mathrm{C} 4 \mathrm{~b}$ (EC4b)- opsonized $\mathrm{E}$ were prepared as described $(3,19)$.

Phagocytosis assays. PMN phagocytosis was assessed by a fluidphase assay as described (1-5). The reaction mixtures are described in the figure legends. Phagocytosis was assessed by light microscopy and quantitated as a phagocytic index (PI), the number of $\mathrm{E}$ ingested/ 100 PMN.

Superoxide anion assay. Superoxide anion was quantitated by cytochrome $c$ reduction as described (4). The data are reported as the superoxide dismutase-inhibitable nanomoles of cytochrome $c$ reduced per $1.0 \times 10^{6} \mathrm{PMN} / 20 \mathrm{~min}$.

Protein kinase $C$ assay. PMN $\left(1.0-1.5 \times 10^{6}\right)$ in $1 \mathrm{ml}$ of $\mathrm{HBSS}^{++}$ were incubated with and without 10 or $100 \mathrm{ng}$ of PMA for $15 \mathrm{~min}$ at $37^{\circ} \mathrm{C}$. The samples were diluted into ice-cold HBSS, pelleted by centrifugation, and resuspended in $1 \mathrm{ml}$ of homogenization buffer, $5 \mathrm{mM}$ Tris, pH 7.5, $1 \mathrm{mM} \mathrm{MgCl} 2,2 \mathrm{mM}$ DTT, $10 \mathrm{mM}$ EGTA, and $10 \mu \mathrm{g} / \mathrm{ml}$ of each leupeptin, aprotinin, pepstatin, and $p$-nitrophenyl $p^{\prime}$-guanidino-benzoate. The suspensions were sonicated for $15 \mathrm{~s}$ on ice before centrifugation at $100,000 \mathrm{~g}$ for $1 \mathrm{~h}$. PKC activity in the supernatant (cytosolic fraction) was assessed as follows: incubation mixtures $(0.25$ $\mathrm{ml}$ ) containing $20 \mathrm{mM}$ Hepes, pH 7.5, $10 \mathrm{mM} \mathrm{MgCl}_{2}, 5 \mathrm{mM}$ DTT, 500 $\mu \mathrm{g} / \mathrm{ml}$ histone III-S (Sigma Chemical Co.), $0.5 \mathrm{mM} \mathrm{CaCl}_{2}, 12 \mu \mathrm{g} / \mathrm{ml}$ 1-oleoyl-2-acetylglycerol, $60 \mu \mathrm{g} / \mathrm{ml}$ phosphatidyl serine (both lipids from Avanti Polar Lipids, Birmingham, AL), $25 \mu$ l of supernatant, $5 \mu 1$ of $\left[\gamma-{ }^{32} \mathrm{P}\right] \mathrm{ATP}(40-50 \mu \mathrm{M}$; New England Nuclear, Boston, MA), and $50 \mu \mathrm{M}$ ATP were incubated for $20 \mathrm{~min}$ at $30^{\circ} \mathrm{C}$. Additional unlabeled ATP was added to a final concentration of $1 \mathrm{mM}$ before application of $10 \mu \mathrm{l}$ of each assay mixture to P81 phosphocellulose paper (Fisher Scientific Co., Pittsburgh, PA). The paper was dried in a microwave oven for $2 \mathrm{~min}$ and, after cooling to room temperature, was washed twice for $20 \mathrm{~min}$ in $10 \%$ TCA containing $10 \mathrm{mM}$ pyrophosphate, and once for $20 \mathrm{~min}$ in 5\% TCA. The paper was rinsed in ethanol (ETOH) 1 min, a 1:1 mixture of ETOH and acetone for $1 \mathrm{~min}$, and finally in acetone for $1 \mathrm{~min}$ before air drying. The radioactivity bound to the paper was assessed by scintillation counting in ScintiVerse (Fisher Scientific Co.). PKC-specific phosphorylation was calculated as the difference between TCA-precipitable radioactivity in complete reaction mixtures and phosphorylation in mixtures lacking $\mathrm{Ca}^{++}$and phosphatidyl serine. PKC activity is reported as the picomoles/minute per $10^{7}$ PMN. Under these reaction conditions, assessment of loss of cytosolic activity was more sensitive and reproducible than was translocation of activity to particulate fractions, presumably because of the rapid cleavage of translocated PKC to a $\mathrm{Ca}^{++}$and phospholipid-independent kinase activity (20).

cAMP assay. PMN $\left(2.0 \times 10^{7} / \mathrm{ml}\right)$ were incubated in $400 \mu \mathrm{M}$ IBMX for 5 min at $37^{\circ} \mathrm{C}$. Without washing, $2 \times 10^{6} \mathrm{PMN}$ were incubated with $20 \mu \mathrm{g}$ of purified antibody in a volume of $150 \mu \mathrm{l}$ for $30 \mathrm{~min}$ at $0^{\circ} \mathrm{C}$. Immune complexes (rabbit IgG anti-BSA/BSA) equivalent to $360 \mu \mathrm{g}$ of antibody $/ \mathrm{ml}$ were added and the mixtures were incubated at $1.0 \times 10^{6} \mathrm{PMN} / \mathrm{ml}$ for $30 \mathrm{~min}$ at $37^{\circ} \mathrm{C}$. In some experiments, FMLP at a final concentration of $1 \mu \mathrm{M}$ was added and the mixtures were incubated for $15 \mathrm{~min}$ at $37^{\circ} \mathrm{C}$. cAMP levels were assessed as described using the acetylation procedure with the radioimmunoassay kit from New England Nuclear (3). Cell supernatants were acetylated and assayed immediately upon generation. Supernatants which were frozen before acetylation had significantly reduced and variable levels of detectable cAMP. The data are represented as net picomoles cAMP per $1.0 \times 10^{7}$ PMN. The averaged value for buffer-treated PMN that was subtracted from the experimental values was $10.1 \pm 0.23 \mathrm{pmol} / 1.0 \times 10^{7} \mathrm{PMN}$.

\section{Results}

LAD PMN fail to ingest IgG- and C4b-opsonized targets (CD11b/CD18-independent opsonins) in response to stimulation. Normal PMN in suspension bind EIgG but are not very efficient at mediating phagocytosis; however, when they are stimulated with an optimal dose of PDBu $(15 \mathrm{ng} / \mathrm{ml})$, ingestion is markedly augmented (references 3 and 4 and Fig. $1 A$ ). This augmentation is reflected not only in the total number of ingested targets (PI) but also in the percentage of phagocytosing PMN ( 30\% for unstimulated PMN vs. $\sim 80 \%$ for PDBustimulated PMN). Unlike ingestion by buffer-treated PMN, ingestion stimulated by PDBu is dependent upon activation of the respiratory burst and is inhibited by the inclusion of catalase in the reaction mixture (Fig. $1 A$, and reference 4). Adhesive proteins containing the sequence RGD also stimulate the ingestion of EIgG; however, they do so by a different mechanism because the inclusion of catalase to inhibit the myeloperoxidase-hydrogen peroxide-halide oxidant system is required to demonstrate RGD-stimulated ingestion (5). As shown in Fig. $1 A$, when normal PMN are stimulated with an optimal dose $(40 \mu \mathrm{g} / \mathrm{ml})$ of a polyvalent RGD ligand (cc-RGDS), ingestion is augmented but only in the presence of catalase. In contrast, PMN from two patients with LAD (Fig. 1, $B$ and $C$ ) failed to respond to an optimal dose of either PDBu or cc-RGDS to augment ingestion either in the presence or absence of catalase. However, ingestion by buffer-treated PMN either in the presence or absence of catalase was the same (Fig. $1 C$ ) or slightly elevated (Fig. $1 B$ ) as compared to normal PMN (Fig. $1 A$ ). In addition, the failure to respond was not simply because of a change in the dose-response curve of the stimulant for LAD PMN, because stimulation of LAD PMN with either PDBu from 15 to $300 \mathrm{ng} / \mathrm{ml}$ (optimal dose $15 \mathrm{ng} / \mathrm{ml}$ ) or cc-RGDS from 20 to $160 \mu \mathrm{g} / \mathrm{ml}$ (optimal dose $40 \mu \mathrm{g} / \mathrm{ml}$ ) still did not result in augmentation of ingestion (data not shown). In addition, the failure of LAD PMN to respond to cc-RGDS was not due to failure to bind the stimulant because we have shown previously that LAD PMN bind equivalent amounts of ccRGDS as compared to normal PMN (5). Two other RGD-containing adhesive proteins, Vn and Fn, also failed to augment ingestion of EIgG by LAD PMN (data not shown). Not only did PDBu stimulation fail to increase ingestion by LAD PMN but it actually decreased the level of ingestion as compared to ingestion by buffer-treated LAD PMN (Fig. 1, $B$ and $C$ ). Similar data were obtained for ingestion stimulated with a low molecular weight cytokine YM-10E (2) and ingestion stimulated with TNF- $\alpha$ (11) (data not shown). A similar observation has been made for the adherence of LAD PMN to vascular endothe-

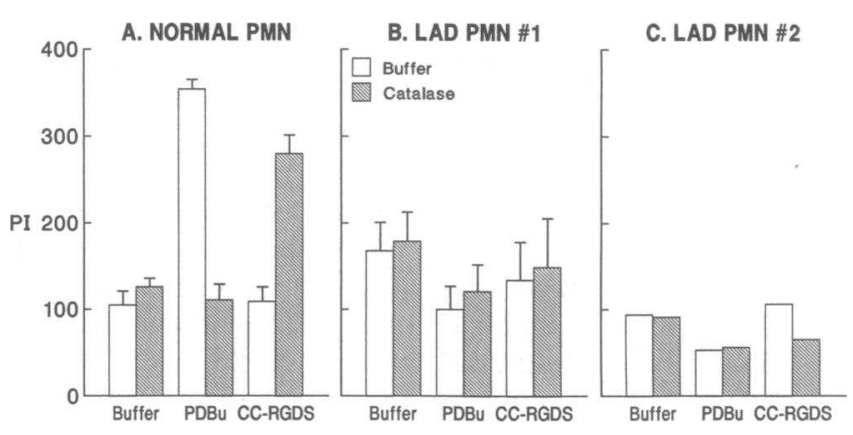

Figure 1. LAD PMN fail to amplify ingestion of EIgG in response to stimulation. PMN $\left(1.0 \times 10^{5}\right)$ from normal controls $(A)$ or two patients with $\operatorname{LAD}(B$, patient 1 and $C$, patient 2 ) were incubated with EIgG in the presence (hatched bars) or absence (open bars) of 5,000 $\mathrm{U}$ of catalase and either buffer, $15 \mathrm{ng} / \mathrm{ml} \mathrm{PDBu}$, or $40 \mu \mathrm{g} / \mathrm{ml}$ of ccRGDS in a final volume of $115 \mu \mathrm{l}$ for $30 \mathrm{~min}$ at $37^{\circ} \mathrm{C}$. Ingestion was quantitated as the PI, that is, the number of EIgG ingested by 100 $\mathrm{PMN}$. Data are represented as the mean $\pm \mathrm{SEM}, n=2-5$ determinations. 
lium: adherence of FMLP-stimulated LAD PMN is decreased as compared to adherence by buffer-treated LAD PMN (21). Identical data as depicted in Fig. 1 were obtained with PMN from a third patient with LAD (data not shown). For our additional studies we have used PDBu stimulation as a model of cytokine-stimulated ingestion and cc-RGDS as a model of extracellular matrix stimulated ingestion to avoid the issue of the failure of the stimulant to bind as an explanation for the failure of LAD PMN to demonstrate stimulated ingestion. However, the failure to demonstrate stimulated ingestion by LAD PMN could be observed with YM-10E, TNF- $\alpha$, Fn, and Vn. Ingestion via another $C D 11 \mathrm{~b} / \mathrm{CD} 18$-independent opsonin, $\mathrm{C} 4 \mathrm{~b}$, also was investigated. As shown in Fig. 2, normal PMN stimulated with an optimal dose of PDBu avidly ingested EC4b. In contrast, PMN from the two LAD patients failed to respond to PDBu to ingest the EC4b. As we have shown previously, the anti-CD11b/CD18 monoclonal Mol is capable of completely abrogating PDBu-stimulated ingestion of EC4b by normal PMN (17). For both EIgG and EC4b, the failure to demonstrate stimulated ingestion was not due to a failure to bind the opsonized particles. PMN from the three patients bound equal numbers of both EIgG and EAC4b as the normal PMN (data not shown). In addition, LAD PMN expressed normal antigenic levels of FcRII, FCRIII, and CR1 as assessed by fluorescent flow microcytometry (data not shown). These data indicate that CD11/CD18-deficient neutrophils fail to respond to pro-inflammatory agents to amplify phagocytic function even for $\mathrm{CD} 1 \mathrm{~b} / \mathrm{CD} 18$-independent ligands.

Ingestion by LAD PMN is PKC-independent. Our previous studies on the molecular mechanisms of IgG-mediated ingestion by non-stimulated and stimulated PMN have led us to hypothesize that ingestion by buffer-treated PMN is independent of PKC activation. In contrast, PMN stimulated with TNF- $\alpha$, granulocyte/macrophage colony-stimulating factor (GM-CSF), or PDBu exhibit ingestion of EIgG which is dependent upon PKC activation (11). Because CD11/CD18-defi-

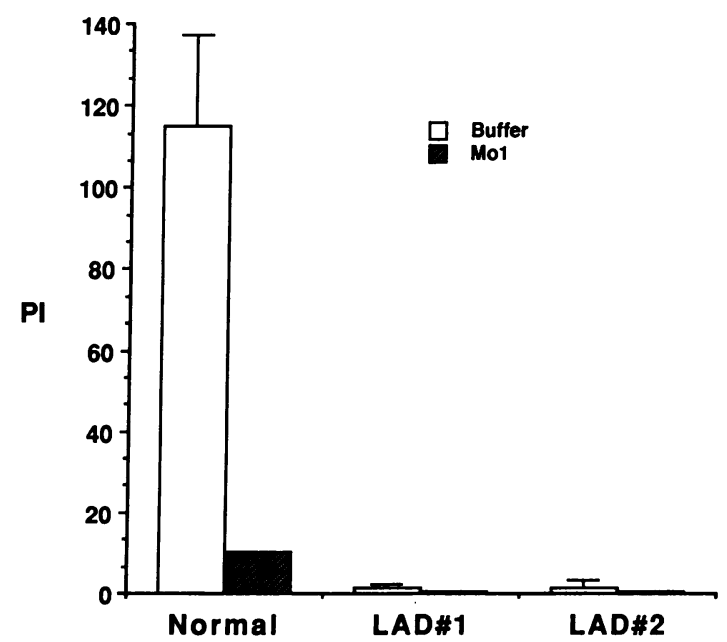

Figure 2. LAD PMN fail to ingest EAC4b in response to stimulation. $\operatorname{PMN}\left(1.0 \times 10^{5}\right)$ from normal controls and two patients with LAD (patient 1 and patient 2) were pretreated with buffer or $0.83 \mu \mathrm{g}$ of purified anti-CD11b/18 (Mol) for $10 \mathrm{~min}$ at room temperature. Without washing, the mixtures were further incubated with EAC4b in the presence of $15 \mathrm{ng} / \mathrm{ml}$ of PDBu in a final volume of $115 \mu \mathrm{l}$ for $30 \mathrm{~min}$ at $37^{\circ} \mathrm{C} . \mathrm{PI}=$ number of EAC4b ingested by $100 \mathrm{PMN}$. Data are represented as the mean \pm SEM, $n=3-6$ determinations. cient $P M N$ failed to respond to activation to enhance ingestion, we sought to investigate the mechanism of ingestion utilized by these PMN as compared to normal PMN by examining the effects of PKC inhibitors and of PKA activators and inhibitors on ingestion by LAD PMN. Possibly, the failure of LAD PMN to respond to stimulation was due to in vivo activation by inflammatory mediators as a result of subclinical infection. As shown in Fig. $3 \mathrm{~A}$, ingestion of EIgG by buffertreated normal PMN was not affected by the isoquinoline sulfonamide derivative $\mathrm{H7}$, a PKC inhibitor, or by a fourfold greater concentration of the control derivative HA1004, which is more specific at this concentration for PKA. In contrast, $\mathrm{PDBu}$ - and cc-RGDS-stimulated ingestion were completely abrogated by $\mathrm{H} 7$ whereas the control HA1004 had no effect (Fig. $3 \mathrm{~A}$ ). In addition, ingestion of EC4b by PDBu-stimulated normal PMN was inhibited $78.3 \%$ by $\mathrm{H} 7$ and was unaffected by HA1004 (data not shown). The inclusion of DiBcAMP inhibited IgG-dependent ingestion by normal PMN, but had no effect on EIgG ingestion stimulated by PDBu or cytokines (Fig. $3 A$ ). This inhibition occurs presumably by activation of PKA and can be overcome by PDBu or cytokine stimulation of normal PMN (Fig. $3 A$ and references 3 and 11). Importantly, ingestion of EIgG by buffer-treated LAD PMN was not inhibited by the inclusion of either $\mathrm{H} 7$ or HA 1004 (Fig. 3 B). Furthermore, DiBcAMP inhibited EIgG ingestion by LAD PMN. Thus, ingestion by buffer-treated normal PMN or LAD PMN is independent of PKC and can be inhibited by PKA, whereas stimulated ingestion, observable only with normal PMN, requires PKC activation. These data demonstrate that the molecular mechanisms used by CD11/CD18-deficient PMN for phagocytosis are similar to those used by unstimulated normal PMN. Therefore, it is unlikely that the failure of LAD PMN to respond to stimulation by $\mathrm{PDBu}$, cytokines, or RGD-containing adhesive proteins reflects an in vivo preactivation to the stimulated state.

The defect in LAD PMN phagocytic function is subsequent to $P K C$ activation. Because the CD18-deficient PMN failed to demonstrate PKC-dependent ingestion in response to phorbol ester stimulation, we investigated the ability of LAD PMN to translocate $\mathrm{PKC}$ from the cytosol in response to activation with phorbol esters. As shown in Fig. 4, LAD PMN lost PKC activity from cytosolic fractions similarly to normal PMN in response to stimulation with increasing concentrations of PMA. Because the LAD PMN could translocate PKC normally, we investigated superoxide anion generation, another cell function thought to be dependent upon PKC activation, to determine whether LAD PMN were defective in all PKC-dependent processes. Several reports examining the generation of superoxide anion by LAD PMN have shown that these cells generate superoxide anion after stimulation with high concentrations of either phorbol esters or FMLP (14). These studies were performed on cells in suspension. However, there has been at least one report of a patient whose PMN in suspension produced elevated levels of superoxide anion as compared to normal PMN in response to FMLP and a reduced response to phorbol ester stimulation (22). In addition, some forms of adherencedependent activation of the respiratory burst appear to be diminished in these patients $(23,24)$. Our studies were performed on cells in suspension under the conditions of the phagocytosis assay. Unlike their complete inability to enhance phagocytosis, both patients' PMN could produce superoxide anion in response to PDBu stimulation (data not shown). PMN from one patient had a significantly heightened response to $P D B u$ and 

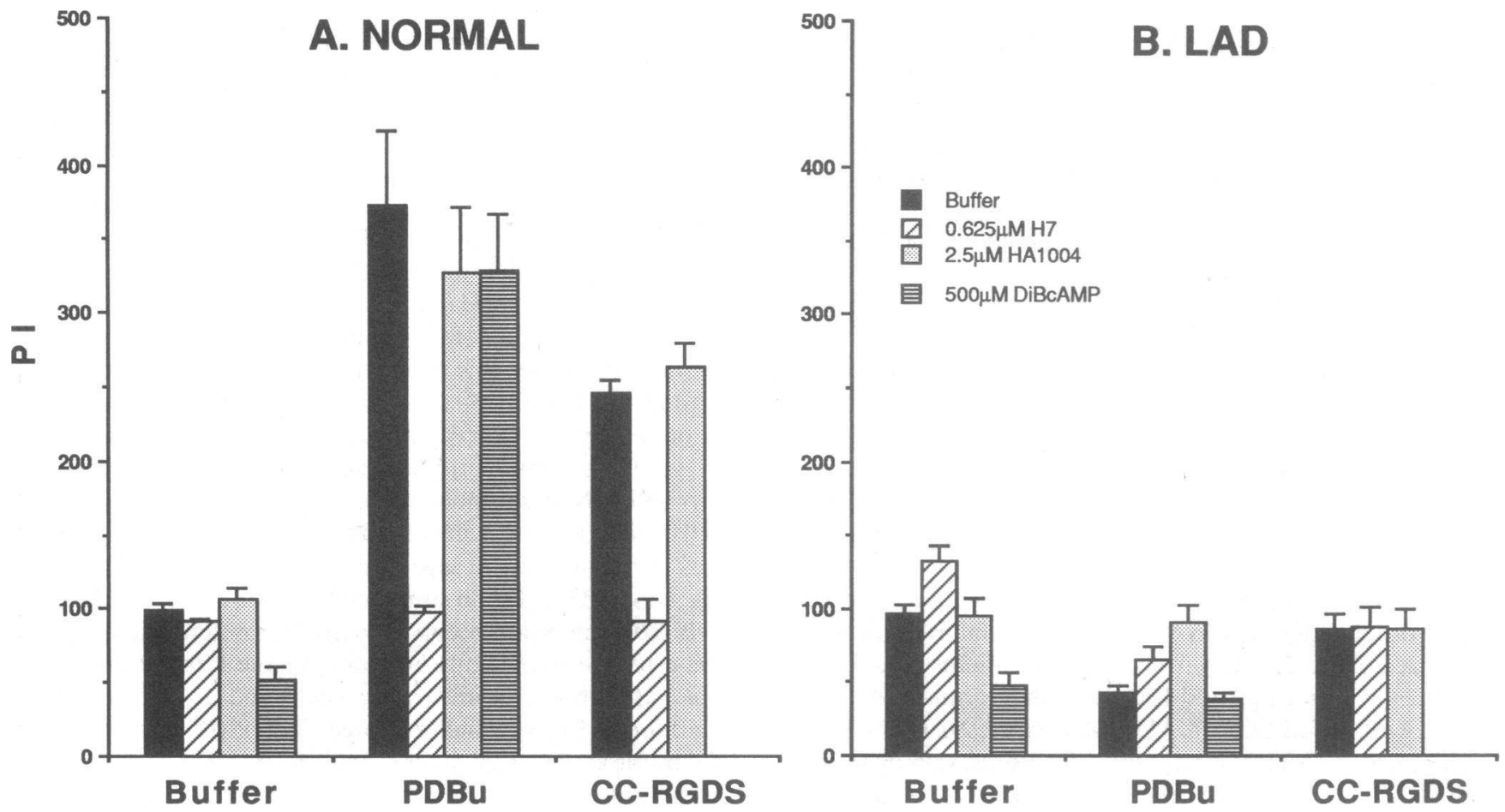

Figure 3. Effect of pharmacologic inhibitors of PKC on ingestion of EIgG by normal PMN and LAD PMN. PMN $\left(1.0 \times 10^{5}\right)$ from normal controls $(A)$ and LAD patients 1 and $3(B)$ were incubated with EIgG in the presence of the indicated pharmacologic agents and either buffer, $15 \mathrm{ng} / \mathrm{ml} \mathrm{PDBu}$, or $40 \mu \mathrm{g} / \mathrm{ml}$ of cc-RGDS in a final volume of $115 \mu \mathrm{l}$ for $30 \mathrm{~min}$ at $37^{\circ} \mathrm{C}$. Tubes containing cc-RGDS also contained 5,000 $\mathrm{U}$ of catalase. PI = number of EIgG ingested by 100 PMN. Data are represented as the mean of duplicate determinations.

the other a slightly diminished response as compared to normal PMN (LAD patient $1,6.41 \pm 0.95 \mathrm{nmol}$ superoxide anion $/ 10^{6}$ PMN; LAD patient 2, 14.2 $\pm 1.6 \mathrm{nmol}$ superoxide anion; and normal PMN, 8.86 \pm 0.95 nmol superoxide anion, mean \pm $\mathrm{SEM}, n=3-4)$. The inclusion of $\mathrm{H} 7$ in the reaction mixtures inhibited superoxide anion generation by LAD and normal PMN equivalently: $47.6 \%$ inhibition of superoxide anion generation by PDBu-stimulated LAD PMN as compared to $56.6 \%$ inhibition for PDBu-stimulated normal PMN (data not shown). Thus, both patients' PMN were able to generate a PKC-dependent respiratory burst. Therefore, these data do not indicate a role for CD1 1/CD18 in superoxide anion generation subsequent to PKC activation as studied in our assay. The variation in the response of the LAD PMN indicates that CD11/ CD18-independent factors (i.e., inflammatory cytokines to which the PMN had been exposed in vivo) may play a role in regulating the magnitude of the response. We conclude that the failure of LAD PMN to recruit PKC-dependent mechanisms for phagocytosis in response to several stimuli does not reflect a general failure to activate PKC or to exhibit PKC-dependent membrane functions.

The defect in LAD PMN phagocytic function is specific for the deficiency of $C D 11 b / C D 18$. Because one of the patients' PMN expressed FCRI even in the absence of evidence of current infection (data not shown), we investigated if the failure of LAD PMN to demonstrate PKC-dependent ingestion was specific for the deficiency of CD11b/CD18 or was due to a nonspecific effect of inflammation. We performed several experiments to address this. First, we examined the ability of PMN from two patients with CGD to demonstrate PKC-dependent ingestion in response to RGD stimulation. We have reported previously that CGD PMN do not demonstrate
PDBu-or cytokine-stimulated ingestion because that stimulation is dependent upon activation of the respiratory burst (4). However, PMN from a CGD patient with a history of recurrent infection similar to the LAD patients increased ingestion of EIgG significantly when stimulated with the RGD-containing adhesive protein, Fn (Fig. 5 B). The PMN from this patient did express a small amount of FcRI (data not shown); this may explain the high level of unstimulated ingestion as compared to unstimulated normal PMN (Fig. 5 A). Even though these PMN exhibited evidence of in vivo inflammatory cytokine activation (25), Fn-stimulated ingestion was still demonstrable. Similar data were obtained with PMN from a second patient with CGD (data not shown). Pretreatment of PMN with the anti-CD18 monoclonal IB4 completely abrogated ingestion by either normal or CGD PMN (Fig. 5). As expected, Fn-stimulated ingestion by CGD PMN, unlike normal PMN, was observable without the inclusion of catalase in the reaction mixture. In addition, when normal PMN were incubated with $100 \mathrm{U} / \mathrm{ml}$ of interferon- $\gamma$ for $6 \mathrm{~h}$ to induce the expression of FcRI (8), they still retained the ability to respond to Fn to enhance ingestion (data not shown). EIgG ingestion by unstimulated PMN increased from a PI $=88 \pm 8$ with control $P M N$ to a $P I=157 \pm 5$ with interferon- $\gamma$-treated PMN. Fn stimulation increased EIgG ingestion for both control $(P I=313 \pm 31)$ and interferon$\gamma$-treated PMN (PI $=332 \pm 49$, mean \pm SEM, $n=3$ ). These data indicate that the defect in phagocytic function observed with LAD PMN is unlikely to be explained by in vivo exposure to inflammatory cytokines which inhibit PKC-dependent ingestion.

Next, we investigated the ability of several MAbs to the CD1 1b/CD18 complex to inhibit only PKC-dependent but not PKC-independent ingestion by normal PMN. We have shown 


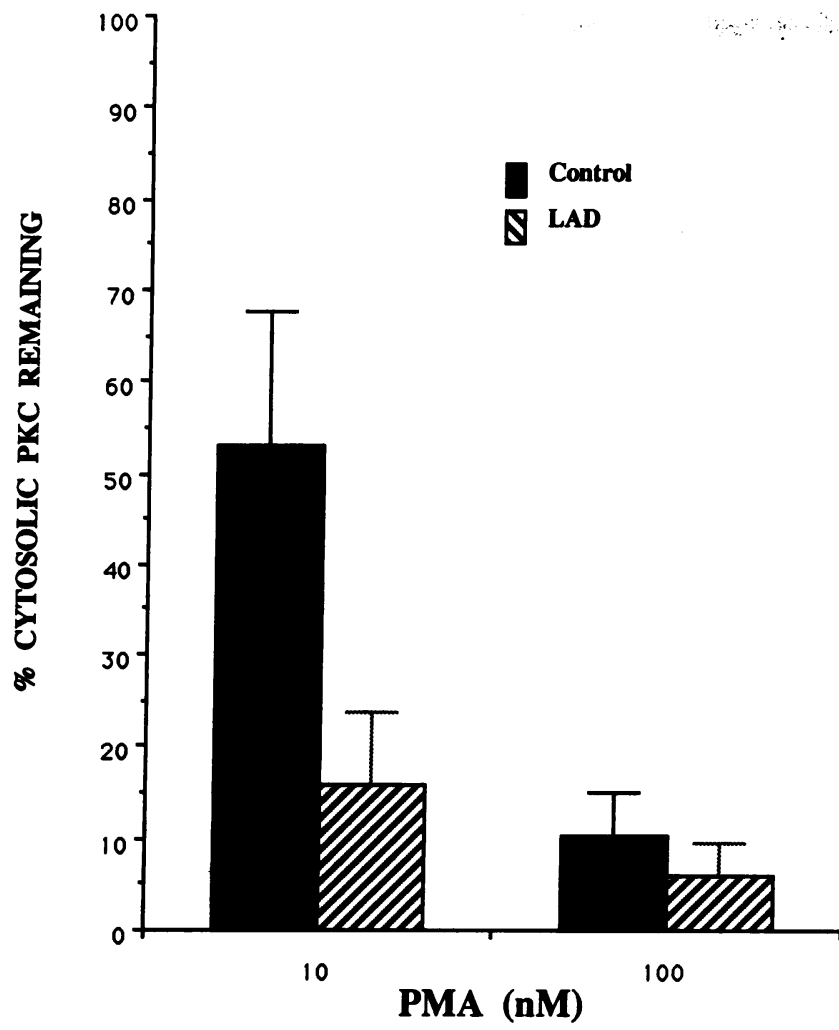

Figure 4. Effect of PMA on cytosolic PKC activity from normal and LAD PMN. PMN (1.0-1.5 $\left.\times 10^{6}\right)$ from normal controls (solid bars) and LAD patient 1 (hatched bars) were stimulated with 10 or $100 \mathrm{ng}$ of PMA in a final volume of $1 \mathrm{ml}$ for $15 \mathrm{~min}$ at $37^{\circ} \mathrm{C}$. The suspensions were sonicated and the PKC activity of the cytosolic fractions was determined as described in Methods. Data represent the mean of duplicate determinations of the percentage of cytosolic PKC activity of buffer controls and are represented as the mean \pm SEM, $n=3$. In a representative experiment, the $100 \%$ cytosolic PKC activity of the buffer control was $4.2 \mathrm{pmol} / \mathrm{min}$ per $10^{7}$ normal PMN and 3.5 $\mathrm{pmol} / \mathrm{min}$ per $10^{7} \mathrm{LAD}$ PMN.

previously that both MAb IB4 and Mol inhibit both unstimulated and stimulated ingestion (17). Therefore, they inhibit both PKC-dependent and -independent ingestion. As shown in Fig. 6, PMN treated with OKM1, a MAb to CD11b, exhibited normal levels of PKC-independent ingestion but failed to respond to either PDBu or Fn to stimulate ingestion, either in the presence or absence of catalase (Fig. $6 \mathrm{~B}$ ). In contrast, PMN treated with an isotype control antibody or with Leu-15, another MAb to CD11b, exhibited both PKC-independent and PKC-dependent ingestion. Increasing the concentration of OKM1 to $10 \mu \mathrm{g} / 1.0 \times 10^{5} \mathrm{PMN}$ (10-fold increase) still had no effect on unstimulated, PKC-independent ingestion by buffertreated normal PMN (data not shown). In addition, a second anti-CD11b MAb, OKM10, gave similar results as OKM1 (data not shown). Thus some MAb against CD11b/CD18 could convert normal PMN to the phenotype of LAD PMN: capable of unstimulated, PKC-independent ingestion, but incapable of any form of PKC-dependent phagocytosis.

The anti-CD18 MAb, IB4, also inhibited PKC-dependent ingestion. However, unlike LAD PMN or OKM1-treated PMN, IB4-treated PMN were also incapable of PKC-independent ingestion of EIgG. We sought to resolve this apparent discrepancy by testing the ability of the PKA inhibitor,

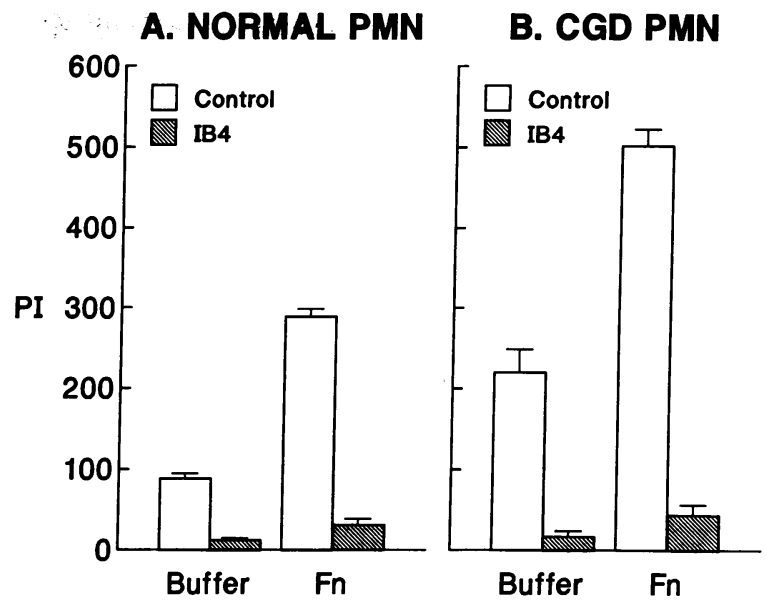

Figure 5. Effect of fibronectin on ingestion of EIgG by CGD and normal PMN. PMN $\left(1.0 \times 10^{5}\right)$ from normal controls $(A)$ or from a patient with CGD $(B)$ were incubated with $1.0 \mu \mathrm{g}$ of purified antiCD18 MAb IB4 (hatched bars) or isotype control (open bars) for 15 min at room temperature. Without washing, the suspensions were further incubated with EIgG in either buffer or $5 \mu \mathrm{g} / \mathrm{ml}$ of Fn for 30 min at $37^{\circ} \mathrm{C}$. Reaction mixtures with normal $\operatorname{PMN}(A)$ also contained $5,000 \mathrm{U}$ of catalase. PI = number of EIgG ingested by $100 \mathrm{PMN}$. Data are represented as the mean \pm SEM, $n=3$.

HA 1004, to reverse IB4-mediated inhibition of EIgG ingestion by buffer-treated PMN. As we have shown previously and in this paper (Fig. 3), activation of PKA by DiBcAMP inhibits PKC-independent ingestion of EIgG. Therefore, we reasoned that MAb IB4 might inhibit ingestion by a similar mechanism. As shown in Fig. $7 A$, PMN treated with MAb IB4, as compared to treatment with a PMN-binding isotype control MAb, were inhibited in their ability to ingest EIgG even when the E were opsonized with a fivefold greater concentration of IgG $\left(\mathrm{EIgG}_{\mathrm{hi}}\right)$. However, when HA1004 was included in the reaction

\section{A. CONTROL ANTIBODY}

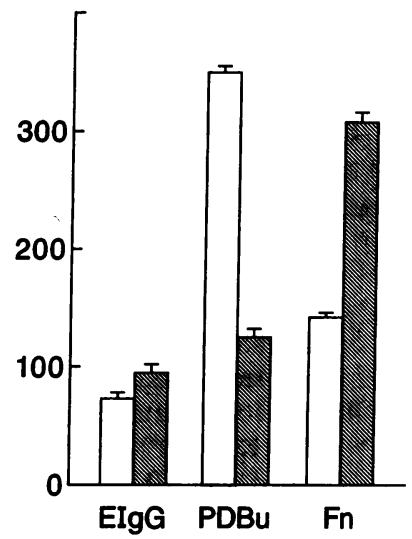

Figure 6. Effect of anti-CD11b/18 monoclonal OKM1 on ingestion of EIgG by normal PMN. PMN $\left(1.0 \times 10^{5}\right)$ were incubated with 1.0 $\mu \mathrm{g}$ of purified isotype control antibody $(A)$ or OKM1 $(B)$ for $1 \mathrm{~h}$ on ice. Without washing, the suspensions were further incubated with EIgG in buffer, $15 \mathrm{ng} / \mathrm{ml}$ of PDBu, or $5 \mu \mathrm{g} / \mathrm{ml}$ of Fn for $30 \mathrm{~min}$ at $37^{\circ} \mathrm{C}$. Reaction mixtures were incubated in the absence (open bars) and in the presence of 5,000 $\mathrm{U}$ of catalase (hatched bars). PI = number of EIgG ingested by 100 PMN. Data are represented as the mean \pm SEM, $n=3$. 
A. BUFFER

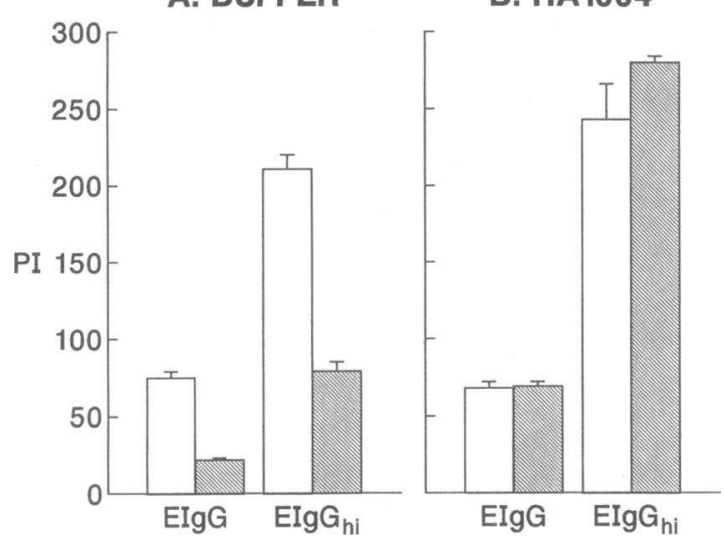

Figure 7. Effect of HA1004 on monoclonal antibody IB4-mediated inhibition of EIgG ingestion. PMN $\left(1.0 \times 10^{5}\right)$ were incubated with $1.0 \mu \mathrm{g}$ of isotype control antibody (open bars) or purified IB4 (hatched bars) for $15 \mathrm{~min}$ at room temperature. Without washing, the suspensions were further incubated with either EIgG or $\mathrm{EIgG}_{\mathrm{hi}}$ in the absence $(A)$ or presence $(B)$ of $5 \mu \mathrm{M}$ HA1004 in a final volume of $115 \mu \mathrm{l}$ for $30 \mathrm{~min}$ at $37^{\circ} \mathrm{C}$. PI = number of EIgG ingested by $100 \mathrm{PMN}$. Data are represented as the mean $\pm \mathrm{SEM}, n=3$.

mixture, treatment of PMN with MAb IB4 no longer inhibited ingestion of either EIgG or $\mathrm{EIgG}_{\mathrm{hi}}($ Fig. $7 \mathrm{~B}$ ). This concentration of HA1004 $(5 \mu \mathrm{M})$ also prevented DiBcAMP-mediated inhibition of ingestion (data not shown). Next, we investigated if HA1004 would also prevent the ability of MAb IB4 to inhibit stimulated or PKC-dependent ingestion. As shown in Fig. 8, MAb IB4 treatment of PMN completely abrogated PDBustimulated ingestion even in the presence of $5 \mu \mathrm{M}$ HA1004. Therefore, inclusion of HA1004 revealed that MAb IB4 could also convert normal PMN to the LAD phenotype, capable of baseline IgG-mediated ingestion but incapable of stimulated ingestion. Identical data were obtained when the effect of HA1004 on Mo1-inhibited ingestion was assessed. The ability of HA1004 to reverse MAb IB4-mediated inhibition of only unstimulated ingestion could not be explained by the absolute level of ingestion involved, because IB4 inhibition of $\mathrm{EIgG}_{\mathrm{hi}}$ ingestion with PI = 240-280 was prevented by HA1004 (Fig. 7 $B$ ) yet IB4 inhibition of PDBu-stimulated ingestion with PI in the same range was unaffected by PKA inhibition (Fig. 8). These data indicate that MAbs Mol and IB4 inhibit PKC-dependent ingestion by a completely different mechanism than

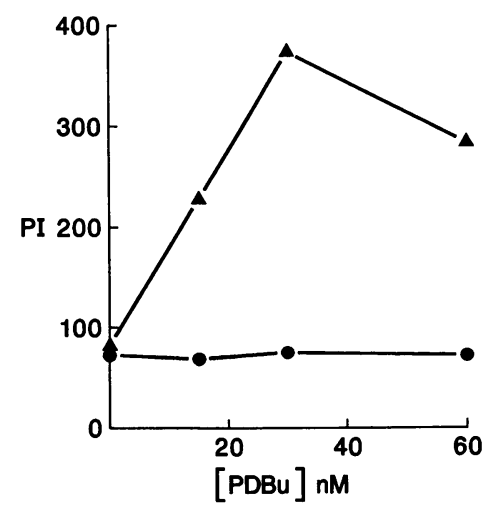

Figure 8. Effect of monoclonal antibody IB4 on PDBu-stimulated ingestion by HA 1004-treated PMN. $\operatorname{PMN}\left(1.0 \times 10^{5}\right)$ were incubated with $1.0 \mu \mathrm{g}$ of isotype control antibody $(\Lambda)$ or purified IB4 (•) for $15 \mathrm{~min}$ at room temperature. Without washing, the reaction mixtures were further incubated with $15 \mu \mathrm{l}$ of

EIgG in the presence of 5 $\mu \mathrm{M}$ HA 1004 and the indicated concentrations of

PDBu. PI = number of EIgG ingested by 100 PMN. Data are representative of two experiments. the mechanism by which they inhibit PKC-independent ingestion of EIgG. Therefore, in the presence of HA1004, IB4 and Mol, as well as OKM 1 and OKM10 convert normal PMN into the phenotype of LAD PMN for phagocytosis.

These data strongly imply that some antibodies against CD11b/CD18 can activate PKA in PMN. To further test this hypothesis we examined the effect of MAbs Mol (antiCD11b) and IB4 (anti-CD18) on intracellular cAMP levels in normal PMN. Leu 15 served as the optimal control antibody for the effects of both Mol and 1B4 because it also binds $\mathrm{CD} 1 \mathrm{~b}$, is the same isotype as Mol and $1 \mathrm{~B} 4$, and does not inhibit either stimulated or unstimulated ingestion of EIgG. As shown in Fig. 9, MAb Mol stimulation of PMN, at the amount of 1 B4 used per PMN in Figs. 7 and $8\left(20 \mu \mathrm{g} / 2.0 \times 10^{6}=1\right.$ $\left.\mu \mathrm{g} / 1.0 \times 10^{5} \mathrm{PMN}\right)$, did not cause a statistically significant increase in intracellular cAMP levels as compared to stimulation with Leu15. In all experiments, cAMP levels of untreated PMN were not significantly different from Leu15-treated PMN. In contrast to incubation in buffer, stimulation with IgG-containing immune complexes (which mimics stimulation by EIgG) resulted in a statistically significant increase in net picomoles cAMP for the Mo1- as compared to the Leu 15treated PMN (Fig. 9). In addition, we assessed the effect of Mol on FMLP-stimulated cAMP accumulation. FMLP was examined because it is known to increase surface Mac-1 by up-regulation of intracellular pools of CD1 lb/CD18. In addition, the effect of FMLP on intracellular levels of CAMP in PMN has been extensively studied (26). FMLP does not stimulate adenylate cyclase directly, but does cause a modest increase in cAMP $\left(\sim\right.$ net $\left.10 \mathrm{pmol} / 10^{7} \mathrm{PMN}\right)$ presumably by inhibition of the cAMP phosphodiesterase (26). As shown in Fig. 9, treatment of PMN with Mol but not Leu 15 resulted in a marked enhancement of cAMP accumulation in FMLP-treated PMN. In fact,

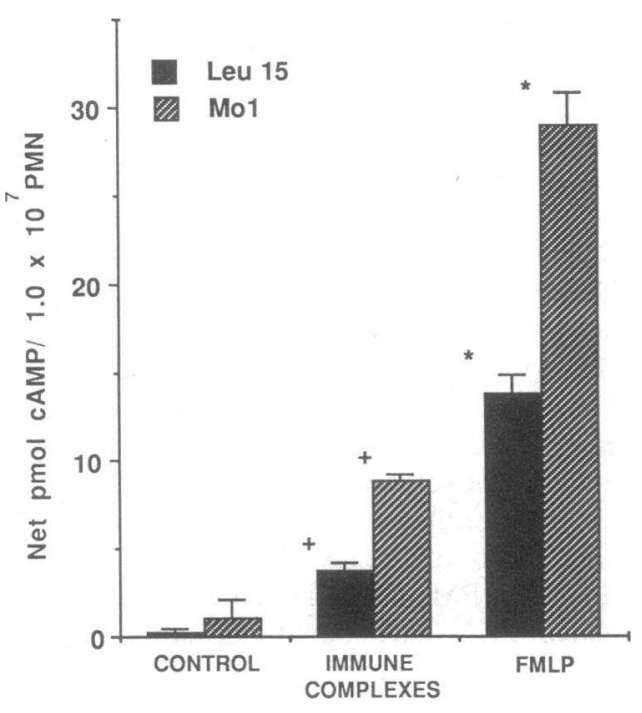

Figure 9. Effect of antibodies Leu15 and Mo1 on net intracellular cAMP levels of unstimulated and stimulated PMN. IBMX-treated PMN $\left(2.0 \times 10^{6}\right)$ were incubated with $20 \mu \mathrm{g}$ of purified Leu 15 or Mol for $30 \mathrm{~min}$ at $0^{\circ} \mathrm{C}$. Either vehicle control, immune complexes at 360 $\mu \mathrm{g}$ of antibody $/ \mathrm{ml}$, or $1 \mu \mathrm{M}$ FMLP was added in a final volume of 2 $\mathrm{ml}$ and incubated at $37^{\circ} \mathrm{C}$. cAMP levels were assessed as described in Methods. Data are represented as the mean \pm SEM net picomoles cAMP $/ 1.0 \times 10^{7} \mathrm{PMN}, n=3 .{ }^{* *} P<0.001$. The averaged value for buffer-treated PMN which was subtracted from the experimental values was $10.1 \pm 0.23 \mathrm{pmol} \mathrm{cAMP} / 1.0 \times 10^{7} \mathrm{PMN}, n=3$. 
Mol treatment doubled the increment in cAMP accumulation as compared to Leu15 treatment of FMLP-stimulated PMN. OKM10 treatment of PMN, which has no effect on unstimulated ingestion, was identical to Leu15 in its effect on cAMP levels (data not shown). In addition to Mo1, 1B4 treatment of PMN as compared to isotype control antibody significantly augmented cAMP levels in response to FMLP stimulation (25.8 \pm 4.2 net pmol cAMP/10 7 PMN with $1 \mathrm{~B} 4$ as compared to $12.1 \pm 5.5$ net pmol cAMP with control antibody, $P<0.01$ ). These experiments were performed on PMN treated with IBMX to inhibit the phosphodiesterase which breaks down cAMP. In previous studies with FMLP, the use of IBMX resulted in an increased sensitivity for the detection of cAMP when examined over time (26). However, in the absence of IBMX, significant increases in CAMP could be detected at earlier time points (26). To assess this, we examined Leu 15- and Mol-treated PMN which had not been treated with IBMX 5 min after stimulation with FMLP. The same twofold increase in net cAMP levels for Mol-treated PMN as compared to control Leu 15-treated PMN was observed (data not shown). Therefore, the use of IBMX did not alter the qualitative assessment of the effect of these antibodies on intracellular cAMP levels. These data indicate that the ability of Mol and 1B4 to inhibit the CD11b/CD18-independent mechanism of ingestion may be due to their effect on the accumulation of cAMP levels in stimulated PMN. In conclusion, the MAb experiments, the studies with CGD PMN, and the studies with IFN- $\gamma$-treated normal PMN demonstrate that the inability of LAD PMN to increase ingestion in response to activation is a direct result of the genetic defect, rather than a consequence of the chronic inflammatory environment to which these PMN are exposed in vivo.

\section{Discussion}

Careful study of phagocytosis over the past few years has suggested that optimal phagocytic function in PMN is recruited at inflammatory sites. Whereas unstimulated ingestion by PMN is a rather inefficient process, ingestion of equivalently opsonized particles can be stimulated by a variety of cytokines and RGD-containing adhesive proteins. Stimulated ingestion differs from unstimulated ingestion not only in the extent and efficiency of the phagocytic process, but also in the molecular pathways involved $(4,11)$. Because cytokines and extracellular matrix proteins are plentiful at inflammatory sites, the phagocytic mechanisms involved in stimulated ingestion are likely to predominate at sites of inflammation and infection. The data presented in this paper demonstrate that, whereas unstimulated phagocytosis is CD11b/CD18 independent, a CD11b/ CD18-dependent mechanism of phagocytosis is recruited in PMN stimulated with multiple pro-inflammatory signals. This is very similar to the recent studies of the mechanisms of adhesion to endothelium used by PMN under various environmental conditions. Whereas adhesion by unactivated PMN is independent of CD1 1b/CD18, FMLP stimulation engages an adhesion mechanism which is dependent on this glycoprotein (21). Thus, activation of PMN recruits a major role for CD11b/ CD1 8 in both adhesion and phagocytosis. Our data on phagocytosis show that the role for CD11b/CD18 is at a step beyond target binding and may be related to an activity for this integrin in the cytoskeletal rearrangements that occur during stimulated ingestion. It is intriguing to speculate that the parallel recruitment of $\mathrm{CD} 11 \mathrm{~b} / \mathrm{CD} 18$ in both adhesion and ingestion by activated PMN suggests a similar non-ligand-binding role for CD1 $1 \mathrm{~b} / \mathrm{CD} 18$ in the spreading of activated PMN on endothelium.

The purpose of the experiments reported here was to determine whether CD11/CD18-deficient PMN from patients with LAD would be capable of recruiting the ingestion mechanisms expected to predominate at inflammatory foci. These studies were performed with targets opsonized with IgG and C4b. These opsonins bind to Fc receptors and CR1 and do not depend on CD11b/CD18 for ligand binding. As reported by others (14), LAD PMN bind EIgG normally and ingest equivalent amounts as normal PMN in the absence of stimulation. However, LAD PMN were completely unresponsive to stimulation with phorbol esters, cytokines, or RGD-containing adhesive proteins (Figs. 1 and 2). This defect was demonstrable for both IgG and C4b opsonins. Thus LAD PMN lacked the ability to respond to inflammatory stimuli to amplify phagocytic function and would be expected to exhibit a marked phagocytic defect at inflammatory sites in vivo.

We examined several alternative hypotheses to explain this defect in LAD PMN phagocytic function. An important concern was that the failure to stimulate ingestion was a result not of the basic genetic defect but secondary to the chronic or recurrent infections which occur in this disease. If the PMN were activated by inflammatory stimuli in vivo, the signals for stimulating ingestion might already have been encountered and the resultant ingestion by unstimulated LAD PMN would use molecular mechanisms similar to in vitro activated normal PMN. Alternatively, the circulating PMN might represent a population which, having been subjected to pro-inflammatory cytokines in vivo, were rendered unresponsive to other stimulatory signals. Neither of these is a likely explanation for our results for several reasons. First, the pattern of pharmacologic inhibition of EIgG ingestion was identical for LAD PMN and unstimulated normal PMN (Fig. 3). Ingestion by unstimulated normal PMN is unaffected by $\mathrm{H} 7$, a PKC inhibitor, but is inhibited by DiBcAMP, whereas phorbol ester-stimulated ingestion exhibits the opposite pattern of inhibition. If LAD PMN were upregulated in vivo, IgG-mediated phagocytosis by these PMN would be inhibited by $\mathrm{H} 7$ but resistant to the effects of $\mathrm{DiB}$ cAMP. In fact, the opposite was the case, demonstrating that LAD PMN use a molecular mechanism for ingestion equivalent to that used by resting, unstimulated, normal PMN.

In addition, other patients with recurrent infections exhibited normal activation for enhanced ingestion in vitro. We examined ingestion by PMN from patients with CGD, another genetic disease with a predisposition to recurrent bacterial infections. These patients represented a population which, like the LAD patients, were subject to chronic or recurrent infections, and the consequent generation of pro-inflammatory cytokines in vivo. Evidence for exposure of both LAD and CGD $P M N$ to inflammatory signals in vivo was given by the variable presence of FcRI on these patients' PMN. Normal resting PMN express minimal amounts of FCRI, but its expression can be induced in vitro by exposure of PMN to interferon- $\gamma(8)$ and it can be detected on the surface of PMN isolated from patients with infectious diseases (25). CGD PMN (Fig. 6) as well as interferon- $\gamma$-stimulated normal PMN (data not shown) were able to increase ingestion of EIgG in response to RGD-containing adhesive proteins, even though they expressed FcRI. These data show that exposure to pro-inflammatory cytokines in vivo did not affect the ability of circulating PMN to respond to stimulatory signals in vitro. Thus, the defect in LAD PMN 
phagocytic function is unlikely to be the consequence of exposure to inflammatory signals in vivo.

If the failure to amplify ingestion in response to stimulation was a consequence of the absence of CD11b/CD18 antigens, then treatment of normal PMN with monoclonal antibodies against $C D 11 \mathrm{~b}$ or CD18 might reproduce the LAD phenotype. Indeed, our data indicate that two MAb recognizing $C D 11 \mathrm{~b}$, OKM1 and OKM10, inhibited the ability of normal PMN to augment ingestion in response to stimulation. Neither MAb affected unstimulated ingestion of EIgG (Fig. 6). Thus OKM1and OKM10-treated normal PMN behaved in vitro like untreated LAD PMN. We asked if this was a property of the particular epitope recognized by these MAbs, because we and others have previously reported that some antibodies (such as IB4 and Mo1) inhibit both unstimulated and stimulated ingestion (PKC independent and dependent) (15-17). We postulated that the antibodies that inhibited both mechanisms of phagocytosis might actually induce the generation of a signal inhibitory to ingestion. The one signal we had determined previously to negatively affect unstimulated ingestion was cAMP, presumably working through PKA (3). When PMN were incubated with HA1004 to inhibit PKA, MAb-IB4 treatment of those PMN no longer inhibited unstimulated ingestion of EIgG, whereas it retained the ability to inhibit PDBu-stimulated ingestion (Figs. 7 and 8). Because MAbs which recognize both CD11b and CD18 could reproduce the LAD defect in vitro, and the defect was not a direct consequence of the in vivo exposure to inflammatory signals, our data indicate that the phagocytic defect in LAD is a direct consequence of the genetic abnormality.

In addition, we assessed the ability of Mol and IB4 to augment intracellular levels of cAMP. Both Mol and 1B4 augmented intracellular cAMP levels in response to stimulation with either immune complexes or FMLP (Fig. 9). Even though most of our experiments were performed with IBMX-treated PMN, identical fold increases in net cAMP could be observed with untreated PMN at earlier time points. Therefore, the effects of Mo1 and 1B4 were not due to an artifact of the IBMX treatment. Our data do not prove directly that Mol and 1B4 inhibit unstimulated ingestion by activation of PKA. Conclusive proof will require elucidation of the mechanism by which cAMP inhibits unstimulated ingestion (i.e., phosphorylation of a specific protein) and showing identical effects of 1B4 and Mo1. Recently published work indicates that 1B4 treatment can increase intracellular cAMP levels by preventing CD11b/ CD18-dependent decreases in cAMP (27). It is possible that Mo1 and 1B4 are working in our studies by a similar passive mechanism. Our data most closely parallel studies performed with anti-CD29 treatment of lymphocytes (28). Increases in intracellular cAMP were observed upon MAb engagement of CD29, a related integrin $\beta$ chain, expressed by lymphocytes, but only when the cells were co-stimulated with anti-CD3 (28). Thus, stimulation of PMN with EIgG in the presence of either Mo1 or 1B4 during the phagocytosis assay could result in local accumulations of cAMP with activation of PKA and generation of a signal inhibitory for PKC-independent ingestion. The significance of this observation to other effects of Mol and 1B4 is unknown; however, it is interesting to speculate that antiCD11b/CD18 activation of PKA may explain some of the anti-inflammatory effects of the anti- $\beta$ chain antibodies in various pathological conditions $(29,30)$. It is equally intriguing to speculate that ligand engagement of $C D 11 \mathrm{~b} / \mathrm{CD} 18$-bearing receptors could also stimulate cAMP accumulation, which may mediate via PKA some of the pleiotropic biological effects of this integrin family.

Because CD11b/CD18-deficient PMN failed to demonstrate PKC-dependent phagocytic mechanisms, we investigated the ability of LAD PMN to activate PKC and to demonstrate other PKC-dependent functions. Using PKC translocation as an assay for PKC activation, we found that phorbol esters translocated PKC normally in LAD PMN (Fig. 4). Thus, failure of PKC translocation could not explain the lack of phagocytic response of LAD PMN to phorbol esters. We also examined the ability of LAD PMN to generate a respiratory burst in response to phorbol esters, another cell function thought to be dependent on PKC activation. We found, as have others, that the generation of superoxide anion in response to phorbol ester stimulation as well as FMLP stimulation occurred but was quantitatively variable and without a consistent pattern of response. Some of the variability may represent differences in prior in vivo exposure to inflammatory signals. Nonetheless, these data showed that there was no general defect in PKC-dependent responses in LAD PMN. Therefore, the inability of LAD PMN to recruit phagocytic mechanisms dependent on PKC activation did not arise from a failure to activate PKC or from a general failure of PKC-dependent signal transduction.

In summary, our data demonstrate a specific defect in phagocytosis by LAD PMN. LAD PMN failed to amplify ingestion in response to stimulation by phorbol esters, cytokines including TNF- $\alpha$, and RGD-containing adhesive proteins. These experiments indicate that a role for $\mathrm{CD} 11 \mathrm{~b} / \mathrm{CD} 18$ is essential for the phagocytic mechanism that predominates at inflammatory foci: i.e., ingestion stimulated by inflammatory signals. Our data indicate that the CD11b/CD18-dependent mechanism of ingestion is also dependent on the activation of PKC. This suggests that PKC may play an important role in the regulation of $\mathrm{CD} 11 \mathrm{~b} / \mathrm{CD} 18$ function, either by direct phosphorylation of the $\beta$ chain (31), or indirectly by phosphorylation of a cytoplasmic protein which alters the conformation or cytoskeletal association of the plasma membrane heterodimer. The importance of PKC-dependent and CD1 1b/CD18-dependent phagocytic mechanisms to host defense is demonstrated by the fact that PMN from patients with diseases with a predisposition to serious recurrent infection (CGD, paroxysmal nocturnal hemoglobinuria, and LAD) fail to exhibit some or all of these mechanisms for the amplification of ingestion. The inability of phagocytes to normally upregulate their ingestion most likely contributes very significantly to the increased propensity to infection in patients with LAD. In support of this possibility is the clinical observation that LAD patients get significant bacterial pneumonias, despite apparently normal ingress of PMN to this site of infection (Dr. Anderson, personal observation).

\section{Acknowledgments}

This work was supported by grants AI-23790 (Dr. Gresham), GM38330 (Dr. Brown), and AI-23521, AI-19031, DE-07875, and HL11422 (Dr. Anderson) from the National Institutes of Health, by a postdoctoral fellowship from the Arthritis Foundation (Dr. Graham), and by the Medical Research Service, Department of Veterans Affairs (Dr. Gresham).

\section{References}

1. Pommier, C. G., J. O'Shea, T. Chused, K. Yancey, M. Frank, and E. J. Brown. 1984. Studies of the fibronectin receptors of human peripheral blood 
leukocytes: morphologic and functional characterization. J. Exp. Med. 159:137151.

2. Gresham, H. D., L. T. Clement, J. E. Lehmeyer, F. M. Griffin, and J. E. Volanakis. 1986. Stimulation of human neutrophil Fc receptor-mediated phagocytosis by a low molecular weight cytokine. J. Immunol. 137:868-875.

3. Gresham, H. D., L. T. Clement, J. E. Volanakis, and E. J. Brown. 1987. Cholera toxin and pertussis toxin regulate the Fc receptor-mediated phagocytic response of human neutrophils in a manner analogous to regulation by monoclonal antibody IC2. J. Immunol. 139:4159-4166.

4. Gresham, H. D., J. A. McGarr, P. G. Shackelford, and E. J. Brown. 1988. Studies on the molecular mechanisms of receptor-mediated phagocytosis: amplification of ingestion is dependent on the generation of reactive oxygen metabolites and is deficient in PMN from patients with chronic granulomatous disease. J. Clin. Invest. 82:1192-1201.

5. Gresham, H. D., J. L. Goodwin, P. M. Allen, D. C. Anderson, and E. J. Brown. 1989. A novel member of the integrin receptor family mediates Arg-GlyAsp-stimulated neutrophil phagocytosis. J. Cell Biol. 108:1935-1943.

6. Wright, S. D., and B. C. Meyer. 1986. Phorbol esters cause sequential activation and deactivation of complement receptors on polymorphonuclear leukocytes. J. Immunol. 136:1759-1764.

7. O'Shea, J. J., E. J. Brown, T. A. Gaither, T. Takahashi, and M. M. Frank 1985. Tumor-promoting phorbol esters induce rapid internalization of the C3b receptor via a cytoskeleton-dependent mechanism. J. Immunol. 135:1325-1330.

8. Perussia, B., M. Kobayashi, M. E. Rossi, I. Anegon, and G. Trichieri. 1987. Immune interferon enhances functional properties of human granulocytes: role of Fc receptors and effect of lymphotoxin, tumor necrosis factor, and granulocyte-macrophage colony stimulating factor. J. Immunol. 138:765-774.

9. Klebanoff, S. J., M. A. Vadas, J. M. Harlan, L. H. Sparks, J. R. Gamble, J. M. Agosti, and A. M. Waltersdorph. 1986. Stimulation of neutrophils by tumor necrosis factor. J. Immunol. 136:4220-4225.

10. Brown, E. J., A. M. Newell, and H. D. Gresham. 1987. Molecular regulation of phagocyte function: evidence for involvement of a GTP binding protein in opsonin-mediated phagocytosis by monocytes. J. Immunol. 139:3777-3782.

11. Gresham, H. D., A. Zheleznyak, J. S. Mormol, and E. J. Brown. 1990. Studies on the molecular mechanisms of human neutrophil Fc receptor-mediated phagocytosis: evidence that a distinct pathway for activation of the respiratory burst results in reactive oxygen metabolite-dependent amplification of ingestion. J. Biol. Chem. 265:7819-7826.

12. Gresham, H. D., and E. J. Brown. 1989. Molecular mechanism of fibronectin-stimulated neutrophil phagocytosis. J. Leukocyte Biol. 46:310. (Abstr.)

13. Anderson, D. C., and T. A. Springer. 1987. Leukocyte adhesion deficiency: an inherited defect in the Mac-1, LFA-1, p150,95 glycoproteins. Annu. Rev. Med. 38:175-194.

14. Anderson, D. C., F. C. Schmalstieg, M. J. Finegold, B. J. Hughes, R. Rothlein, L. D. Miller, S. Kohl, M. F. Tosi, R. L. Jacobs, T. C. Waldrop, et al. 1985. The severe and moderate phenotypes of heritable Mac-1, LFA-1, p150,95 deficiency: their quantitative definition and relation to leukocyte dysfunction and clinical features. J. Infect. Dis. 152:668-689.

15. Arnaout, M. A., R. F. Todd III, N. Dana, J. Melamed, S. F. Schlossman, and $H$. R. Colten. 1983. Inhibition of phagocytosis of complement C3- or immunoglobulin G-coated particles and C3bi binding by monoclonal antibodies to a monocyte-granulocyte membrane glycoprotein (Mo1). J. Clin. Invest. 72:171179.

16. Brown, E. J., J. F. Bohnsack, and H. D. Gresham. 1988. Mechanism of inhibition of immunoglobulin G-mediated phagocytosis by monoclonal antibodies that recognize the Mac-1 antigen. J. Clin. Invest. 81:365-375.

17. Graham, I. L., H. D. Gresham, and E. J. Brown. 1989. An immobile subset of plasma membrane $\mathrm{CD} 1 \mathrm{lb} / 18(\mathrm{Mac}-1)$ is involved in phagocytosis of targets recognized by multiple receptors. J. Immunol. 142:2352-2358.

18. Bohnsack, J. F., T. Takahashi, and E. J. Brown. 1986. Interaction of culture-derived macrophages with the fibroblast-binding domain of fibronectin is a necessary but inefficient signal for fibronectin enhancement of CR1-mediated phagocytosis. J. Immunol. 136:3793-3798.

19. Bohnsack, J. F., H. K. Kleinman, T. Takahashi, J. J. O'Shea, and E. J. Brown. 1985. Connective tissue proteins and phagocytic cell function. $J$. Exp. Med. 161:912-923.

20. Melloni, E., S. Pontremoli, M. Michetti, O. Sacco, B. Sparatore, and B. L. Horecker. 1986. The involvement of calpain in the activation of protein kinase $\mathrm{C}$ in neutrophils stimulated by phorbol myristic acid. J. Biol. Chem. 261:41014105 .

21. Smith, C. W, S. D. Marlin, R. Rothlein, C. Toman, and D. C. Anderson. 1989. Cooperative interactions of LFA-1 and Mac-1 with intercellular adhesion molecule- 1 in facilitating adherence and transendothelial migration of human neutrophils in vitro. J. Clin. Invest. 83:2008-2017.

22. Nauseef, W. M., P. de Alarcon, J. F. Bale, and R. A. Clark. 1986. Aberrant activation and regulation of the oxidative burst in neutrophils with Mol glycoprotein deficiency. J. Immunol. 137:636-642.

23. Nathan, C., S. Srimal, C. Farber, E. Sanschez, L. Kabbash, A. Asch, J. Gailit, and S. D. Wright. 1989. Cytokine-induced respiratory burst of human neutrophils: dependence on extracellular matrix proteins and CD11/CD18 integrins. J. Cell Biol. 109:1341-1349.

24. Shappell, S. B., C. Toman, D. C. Anderson, A. A. Taylor, and C. W. Smith. 1989. Adherence-dependent neutrophil hydrogen peroxide secretion is mediated by Mac-1 (CD11b/18). J. Immunol. 144:2702-2711.

25. Simms, H. H., M. M. Frank, T. C. Quinn, S. Holland, and T. A. Gaither. 1989. Studies on phagocytosis in patients with acute bacterial infections. J. Clin. Invest. 83:252-260.

26. Verghese, M. W., K. Fox, L. C. McPhail, and R. Snyderman. 1985. Chemoattractant-elicited alterations in cAMP levels in human polymorphonuclear leukocytes require a $\mathrm{Ca}^{2+}$-dependent mechanism which is independent of trans membrane activation of adenylate cyclase. J. Biol. Chem. 260:6769-6775.

27. Nathan, C., and E. Sanchez. 1990. Tumor Necrosis Factor and CD11/ CD18 integrins act synergistically to lower cAMP in human neutrophils. J. Cell Biol. 111:2171-2181.

28. Groux, H., S. Ituet, H. Valentin, D. Pham, and A. Bernard. 1989. Suppressor effects and cyclic AMP accumulation by the CD29 molecule of CD4+ lymphocytes. Nature (Lond.). 339:152-154.

29. Vedder, N. B., R. K. Winn, C. L. Rice, E. Y. Chi, K.-E. Arfors, and J. M. Harlan. 1988. A monoclonal antibody to the adherence-promoting leukocyte glycoprotein, CD18, reduces organ injury and improves survival from hemorrhagic shock and resuscitation in rabbits. J. Clin. Invest. 81:939-944.

30. Tuomanen, E. I., K. Saukkonen, S. Sande, C. Cioffe, and S. D. Wright. 1989. Reduction of inflammation, tissue damage, and mortality in bacterial meningitis in rabbits treated with monoclonal antibodies against adhesion-promoting receptors of leukocytes. J. Exp. Med. 170:959-968.

31. Chatila, T. A., R. L. Geha, and M. A. Arnout. 1989. Constitutive and stimulant-induced phosphorylation of CD11/CD18 leukocyte adhesion molecules. J. Cell Biol. 109:3435-3444. 